\title{
Increased odds ratio for erectile dysfunction in COVID-19 patients
}

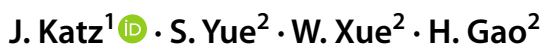 \\ Received: 25 August 2021 / Accepted: 21 November 2021 / Published online: 30 November 2021 \\ (C) Italian Society of Endocrinology (SIE) 2021
}

\begin{abstract}
Purpose Erectile dysfunction and COVID-19 share similar risk factors, including vascular disruption of integrity, cytokine release, cardiovascular disease, diabetes and obesity.

The aim of this study was to investigate the association between erectile dysfunction and COVID-19 patients.

Methods Odds ratio for erectile dysfunction in patients with a history of COVID-19 with and without comorbidities were calculated using a patients' registry platform $\mathrm{i} 2 \mathrm{~b} 2$. ICD-10 diagnoses codes were accessed for queries and data were analyzed using logistic regression.

Results Patients with COVID-19 were 3.3 times more likely to have erectile dysfunction with $95 \%$ CI $(2.8,3.8)$. The association became stronger with odds ratio $4.8(95 \% \mathrm{CI}(4.1,5.7))$ after adjusting for age groups. The odds ratio remained the same after adjusting for smoking status with 3.5 (95\% CI $(3.0,4.1)$ ). After adjusting for race, COVID-19 patients were 2.6 $(95 \%$ CI $(2.2,3.1))$ times more likely to have erectile dysfunction. The odds ratio were 1.6, 1.8, 1.9 and 2.3 after adjusting for respiratory disease, obesity, circulatory disease and diabetes, respectively.

Conclusion COVID-19 and erectile dysfunction are strongly associated even after adjustment for known risk factors and demographics.
\end{abstract}

Keywords Erectile dysfunction $\cdot$ COVID-19 $\cdot$ Odds ratio $\cdot$ Endothelial disruption

\section{Introduction}

Erectile dysfunction is a multidimensional common male sexual dysfunction that includes organic, relational and psychological factors. Roles for nonendocrine and endocrine pathways have been suggested with proposed roles for metabolic syndrome and cardiovascular disease [1].

The risk factors for erectile dysfunction (ED) and COVID-19 infection share striking similarities. Cardiovascular diseases, obesity, diabetes, vitamin D deficiency and generalized anxiety have been all strongly associated with ED [1-3], and with COVID-19 [4-7]. In addition,

J. Katz

jkatz@dental.ufl.edu

1 Department of Oral and Diagnostic Sciences, University of Florida College of Dentistry, POB 100414-0414, Gainesville, FL 32610, USA

2 Department of Biostatistics, College of Public Health and Health Professions, University of Florida, Gainesville, USA
African-American and smokers have been reported to be disproportionally impacted by both conditions $[8,9]$

Since damaged vascular integrity, compromised endothelium and cytokines flush, are common features for both ED and COVID-19 infection [10-13] and may underline the progression of mechanisms of complications in both diseases. We have hypothesized that people affected by COVID-19 have an increased risk for ED as their endothelium has been compromised by the infection. Review of the current literature has identified one publication that has raised the plausibility of the association [14]. Recently, one study used a validated questionnaires and complex statistical analysis to show increased risk of ED in COVID-19 patients [15]. Another study reported on histopathological, immunohistochemical, and ultrastructural findings on penile tissue of COVID-19 patients. The authors have demonstrated the presence of the COVID-19 virus in the penis long after the initial infection in humans and suggest that widespread endothelial cell dysfunction from COVID-19 infection can contribute to ED [16].

The testis, sperm and testosterone levels and vascular bed are all affected by COVID-19 [17] and are directly 
implicated in ED [18]. At the level of testicular tissues, cells have a higher rate of mRNA expression in ACE2 [19]. The testicular expression of ACE2 is age-related and the highest expression of ACE2 has been recorded in younger groups than old [20]. Therefore, the SARS-CoV-2 virus may inflicts detrimental effects on the vascular and endocrine health resulting in erectile dysfunction.

The purpose of the present study was to investigate the association between COVID-19 confirmed patients and ED using a cross-sectional design enabled by a cohort of patients in a large Health Center.

\section{Materials and methods}

The aggregated data extracted from Informatics for Integrating Biology and the Bedside (i2b2), http://www.i2b2. org) is an NIH-supported National Center for Biomedical Computing that developed a scalable informatics framework designed for translational research with collaboration of the University of Florida (UF). The i $2 \mathrm{~b} 2$ reports based on electronic health records (HER) were used in logistics regressions. We have used the international coding of diseases (ICD) 10 platform for our queries. The ICD-10 is the recent system used by physicians and other health care providers in the USA to classify and code of all diagnoses, symptoms, medications and procedures recorded in conjunction with a hospital care.

The study population included patients attending the different UF Health centers across the state of Florida in the period of January 2020-June 2021. The electronic records (EPIC) were searched for diagnostic codes ICD-10. ED, N52, cardiovascular diseases (CVD0, 10 I00-199, diabetes Meletus (DM), E08-E13, and generalized anxiety F41.9. The number of positive COVID-19 patients, patients on testosterone or on the PDE5 inhibitors tadalafil, sildenafil, vardenafil, avanafil was provided by the Integrated Data Repository (IDR).

ED diagnoses were made by the treating physicians based on clinical evaluation.

The IDR consists of a secure, clinical data warehouse that aggregates figures from the UF Health various clinical and administrative information systems, including the Epic electronic health record system. The IDR contains more than 1 billion observational facts pertaining to more than 1 million patients.

The IDR also provided the total number of patients, their age and sex distribution and smoking status. In all cases, the occurrence of COVID-19 preceded the diagnosis of ED. We have performed the searches when the first event of COVID19 diagnosis was preceding the first event of ED diagnosis. The study was exempted by the UF Health Center Institutional Review Board (IRB) as it did not include personal health information (PHI). Logistic regressions were conducted to investigate the associations between ED and other variables of interest. The aggregated counts generated from I2B2 data (instead of individual health records) were used in logistic regressions. Note that fitting logistic regression on aggregate/count data is no different from fitting logistic regression on standard data, given that the aggregated data can always be expanded into the standard form where each row represents an individual record [21]. Therefore, it can be viewed as a standard logistic regression. However, limited by I2b2 data, we can only adjust for one covariate at a time. Respiratory disease, obesity, diabetes, circulatory disease, anxiety, smoking status, and demographics (gender, age groups, and race) was adjusted as covariates one at a time. SAS 9.4, procedure logistic was used to perform the primary analysis.

To investigate the odds ratio for ED for patients on PDE5 inhibitors and testosterone as well as to determine prevalence of ED in post COVID -19 patients without circulatory diseases, we used the MedCalc Software Ltd. Odds ratio calculator. https://www.medcalc.org/calc/odds_ratio.php (Version 20.011; accessed September 21, 2021).

\section{Results}

From a total population of 1,066,108 patients, 9554 patients were diagnosed with ED. The total number of COVID-19 patients was 7098 (3098 were males), and 146 were diagnosed both with ED and COVID-19 (4.7\% of the COVID19 male population). In all cases, the COVID-19 diagnosis has preceded the diagnosis of ED. Patients with COVID-19 were 3.68 times more likely to have ED than patients without COVID-19 (95\% CI 3.33-4.05; $P<0.0001$ ).

The association remained significant after adjusting for age group (OR 5.27; 95\% CI 4.77-5.81; $P<0.0001$ ), and anxiety (OR 2.98; 95\% CI 2.70-3.29; $P<0.0001$ ).

Age group and anxiety were both significantly associated with $\mathrm{ED}(P<0.0001)$. Note that the risks for ED of patients in the age group 45-64 were almost the same as those of patients in the age group $>65$ (OR 0.99 ; 95\% CI 0.96-1.03; $P>0.05)$.

The odds ratio remain the same after adjusting for smoking status with 3.5 (95\% CI $(3.0,4.1)$ ). After adjusting for race (white or African- American), COVID-19 patients are $2.6(95 \%$ CI $(2.2,3.1))$ times more likely to have ED. The odds ratio is $1.6,1.8,1.9$ and 2.3 after adjusting for respiratory disease, obesity, circulatory disease and diabetes, respectively (Table1).

The prevalence of ED in the patients with a history of COVID-19, total hospital population and patients with COVID-19 History but without circulatory disease was $4.67 \%, 1.7 \%$ and $2.7 \%$ respectively. 
Table 1 Odds ratio for Erectile dysfunction for patients with a history of COVID - 19 before and after adjustment for age, race and comorbidities

\begin{tabular}{|c|c|c|c|c|}
\hline \multirow[b]{2}{*}{ History of COVID-19 vs. no history before adjustments } & \multirow{2}{*}{$\begin{array}{c}\text { Odds ratio } \\
3.68\end{array}$} & \multicolumn{2}{|c|}{$\begin{array}{l}\text { 95\% wald confi- } \\
\text { dence limits }\end{array}$} & \multirow{2}{*}{$\begin{array}{l}P \text { value } \\
<0.0001\end{array}$} \\
\hline & & 3.33 & 4.05 & \\
\hline History of COVID-19 vs. no history & 5.27 & 4.77 & 5.81 & $<0.0001$ \\
\hline Age $35-44$ vs $<34$ & 5.21 & 4.65 & 5.83 & $<0.0001$ \\
\hline Age $45-65$ vs $<34$ & 13.18 & 12.04 & 14.46 & $<0.0001$ \\
\hline Age $>65$ vs $<34$ & 13.10 & 11.97 & 14.37 & $<0.0001$ \\
\hline History of COVID-19 vs. no history & 5.27 & 4.77 & 5.81 & $<0.0001$ \\
\hline Age $35-44$ vs $<34$ & 5.21 & 4.65 & 5.83 & $<0.0001$ \\
\hline Age $45-65$ vs $35-44$ & 2.53 & 2.34 & 2.74 & $<0.0001$ \\
\hline Age $>65$ vs $45-65$ & 0.99 & 0.96 & 1.03 & 0.7735 \\
\hline History of COVID-19 vs. no history & 2.626 & 2.229 & 3.093 & $<0.0001$ \\
\hline Race AA vs other & 8.978 & 8.273 & 9.743 & $<0.0001$ \\
\hline Race W vs other & 7.129 & 6.637 & 7.658 & $<0.0001$ \\
\hline History of COVID-19 vs. no history & 2.98 & 2.70 & 3.29 & $<0.0001$ \\
\hline Anxiety vs. No Anxiety & 3.70 & 3.53 & 3.88 & $<0.0001$ \\
\hline History of COVID-19 vs. no history & 1.862 & 1.572 & 2.206 & $<0.0001$ \\
\hline Circulatory disease vs. no circulatory disease & 16.815 & 16.043 & 17.623 & $<0.0001$ \\
\hline History of COVID-19 vs. no history & 1.55 & 1.311 & 1.834 & $<0.0001$ \\
\hline Respiratory disease vs. no respiratory disease & 5.559 & 5.337 & 5.789 & $<0.0001$ \\
\hline History of COVID-19 vs. no history & 3.506 & 2.972 & 4.136 & $<0.0001$ \\
\hline Smoking vs. no smoking & 4.64 & 4.456 & 4.831 & $<0.0001$ \\
\hline History of COVID-19 vs. no history & 1.796 & 1.517 & 2.127 & $<0.0001$ \\
\hline Obesity vs. no obesity & 7.921 & 7.587 & 8.27 & $<0.0001$ \\
\hline History of COVID-19 vs. no history & 2.264 & 1.911 & 2.682 & $<0.0001$ \\
\hline Diabetes mellitus vs. no diabetes mellitus & 9.273 & 8.869 & 9.695 & $<0.0001$ \\
\hline
\end{tabular}

AA African-American, $W$ White
History of COVID-19 was negatively associated with the use of testosterone and PDE5 inhibitors (Table 2).

As registry studies are often prone to error we ran a query on the number of women in the population under ED (ICD-10) N52. No females were identified.

\section{Discussion}

In the present cross-sectional retrospective study, we have found that patients with COVID-19 are 3.3 times more likely to have ED with $95 \% \mathrm{CI}(2.8,3.8)$. The association becomes stronger with OR 4.8 (95\% CI $(4.1,5.7))$ after adjusting for age groups. The association was still robust after we adjusting for cardiovascular, respiratory, DM, race and smoking.

Table 2 Odds ratio for use of PED5 inhibitors in patients with a history of COVID-19 during pandemic period

\begin{tabular}{llllll}
\hline & Patients with ED & Users of PED5 inhibitors & $\begin{array}{l}\text { Users of Testosterone } \\
\text { replacement therapy }\end{array}$ & $\begin{array}{l}\text { Patients with ED with } \\
\text { no circulatory disease }\end{array}$ & \multicolumn{1}{c}{ Hospital } \\
History of COVID-19 & $528(4.67 \%)^{\mathrm{a}}$ & $558(2.32) \%$ & $187(2) \%$ & $76(2.7 \%)^{\mathrm{c}}$ & 2765 \\
Hospital & $11,283(1.7 \%)^{\mathrm{b}}$ & 23,983 & 9146 & 631,034 \\
Odds ratio & & 0.7885 & 0.72 & \\
$95 \%$ CI & & 0.7230 to 0.8599 & 0.6213 to 0.8313 & \\
$p$ & & $<0.0001$ & $<0.0001$ & \\
\hline
\end{tabular}

${ }^{\text {a }}$ Prevalence of ED in patients with a history of COVID-19

${ }^{b}$ Prevalence of ED in the total hospital population

${ }^{\mathrm{c}}$ Prevalence of ED in patients with a history of COVID-19 with no circulatory disease 
The interpretation of cross-sectional study cannot include causality, however, it gives an opportunity to investigate the strength of association between the two investigated conditions [22].

Ample evidence supports the notion that erectile function can be used as a marker of systemic health in general, and vascular performance in particular [23] and that erectile function can be a predictor of heart disease [24]. Vascular integrity is necessary for ED [1], and vascular damage associated with COVID-19 is likely to affect the compromised vascular bed of the penis, resulting in impaired erectile function [25]. SARS-CoV-2 infection can significantly affect the heart and exacerbate underlying cardiovascular conditions. Reports of myocarditis in COVID-19 patients have been accumulating recently [26]. Similarly, arrhythmias and acute cardiovascular events have been described in COVID-19 [25]. COVID-19 survivors are, therefore, more likely to develop severe cardiovascular consequences and consecutively ED. Recent evidences suggested that men may be considered as at higher risk of poor prognosis or death once the infection occurred and concerns surfaced in regard of the risk of a possible testicular injury due to SARS-CoV-2 infection [27].

A literature research on the possible mechanisms involved in the development of ED in COVID-19 survivors was recently performed [14]. The study authors summarized that endothelial dysfunction, subclinical hypogonadism, psychological distress and impaired pulmonary hemodynamics, all contribute to the potential onset of ED. According to the authors, COVID-19 might exacerbate cardiovascular conditions; therefore, further increasing the risk of ED.

Furthermore, while it has been confirmed that SARSCoV-2 could be spread by human-to-human transmission, mainly through respiratory droplets and contact several studies have suggested that the SARS-CoV-2 is found in the semen and testicle in men infected by SARS-CoV-2 at both acute and recovery phases [28].

SARS-CoV-2 binds to the ACE2 receptors and activates the cellular serine protease (TMPRSS2) for S protein priming [29]. ACE2 receptors are established at higher concentrations in the teste and on developing sperm and are involved in production of testosterone, the male sex hormone. High ACE2 expression in testis (both germ cells and somatic cells) suggest potential tropism of COVID-19 to testicular tissues [30].

Lower levels of serum testosterone (total and free) act as predictors of poor prognosis in SARS-CoV-2 men [31] It is not clear whether this hypogonadism is permanent or temporary. Testosterone acts as a modulator for endothelial function [32], and suppresses inflammation by increasing levels of anti-inflammatory cytokines (such as IL-10) and reducing levels of pro-inflammatory cytokines such as TNF- $\alpha$, IL-6 and IL-1 $\beta$ [33]. Therefore, it can be hypothesized that suppression of testosterone levels might be one of the reasons for the large difference in terms of mortality and hospitalization rate between males and females and might also explain why SARS-CoV-2 most commonly infects old men.

Psychological/psychiatric conditions, such as anxiety and depression, which can also contribute to the pathogenesis of ED in the general population-and possibly even more in COVID-19 patients should be considered while evaluating risk factors for ED [7, 34]. In addition, Mollaioli et al. have demonstrated that COVID-19 lockdown dramatically impacted on psychological, relational, and sexual health of the population while sexual activity played a protective effect [35]. To verify whether the ED and COVID -19 association is entirely related to the anxiety and depression associated with the pandemic, we have run an additional computation of the odds ratio while excluding patients that were diagnosed with non-specific anxiety disorder at the time of the pandemic. Although the OR decreased the association remained still robust and statistically significant.

ED is largely known to be an under-reported issue for men due to stigma, shame and lack of awareness. For this reason, we have investigated how many individuals were prescribed PDE5 inhibitors (such as sildenafil or tadalafil) or testosterone therapy following the onset of COVID-19. We did not identify an increased use of these therapies in the ED with positive COVID-19 group. We speculate that the short time elapsed from the beginning of the pandemic in January 2020 is not sufficient to assess whether the use of such drugs might be a surrogate marker of ED in affected individuals.

Our study suffers from few weaknesses. The i2b2 platform uses a patients' registry platform without a direct access to the individual data that includes all their medical history. In addition, we could not adjust for all the comorbidities at the same time but only one at the time. We were not able to determine whether and to what extent COVID-19 is able to impair sexual and reproductive function more than other serious systemic diseases. This study could not assess the severity of COVID-19 in the study population this introduces a not insignificant source of bias and heterogeneity when the outcome is erectile function. Although the authors have performed regression analyses adjusting for a number of possible confounding factors, the probability that key uncontrolled confounders' escape evaluation remains high.

Cross-sectional studies are carried out to investigate associations between risk factors and the outcome of interest and do not infer causality [22].

Furthermore, longitudinal and prospective studies are warranted to determine causality and direction of the association. 


\section{Declarations}

Conflict of interest The authors declare that they have no conflicts of interest.

\section{References}

1. Yafi FA, Jenkins L, Albersen M, Corona G, Isidori AM, Goldfarb S, Maggi M, Nelson CJ, Parish S, Salonia A, Tan R, Mulhall JP, Hellstrom WJ (2016) Erectile dysfunction. Nat Rev Dis Primers 2:16003. https://doi.org/10.1038/nrdp.2016.3 (PMID: 27188339; PMCID: PMC5027992)

2. Imprialos KP, Stavropoulos K, Doumas M, Tziomalos K, Karagiannis A, Athyros VG (2018) Sexual dysfunction, cardiovascular risk and effects of pharmacotherapy. Curr Vasc Pharmacol 16(2):130-142. https://doi.org/10.2174/15701611156661706091 01502 (PMID: 28595561)

3. Weir EK, Thenappan T, Bhargava M, Chen Y (2020) Does vitamin D deficiency increase the severity of COVID-19? Clin Med (Lond) 20(4):e107-e108. https://doi.org/10.7861/clinmed.-0301 (Epub 2020 Jun 5. PMID: 32503801; PMCID: PMC7385774)

4. Guo T, Fan Y, Chen M, Wu X, Zhang L, He T, Wang H, Wan J, Wang X, Lu Z (2020) Cardiovascular implications of fatal outcomes of patients with coronavirus disease 2019 (COVID-19). JAMA Cardiol 5(7):811-818. https://doi.org/10.1001/jamacardio. 2020.1017 (Erratum in: JAMA Cardiol. 2020 Jul 1;5(7):848. PMID: 32219356; PMCID: PMC7101506)

5. Katz J, Yue S, Xue W (2020) Dental diseases are associated with increased odds ratio for coronavirus disease 19. Oral Dis. https://doi.org/10.1111/odi.13653 (Epub ahead of print. PMID: 32989904; PMCID: PMC8004536)

6. Wei Y, Chen P, Chen Q, Zhu H (2019) Serum vitamin D levels and erectile dysfunction: a systematic review and meta-analysis. Andrologia 51(3):e13211. https://doi.org/10.1111/and.13211 (Epub 2018 Dec 6. PMID: 30523636)

7. Omar SS, Dawood W, Eid N, Eldeeb D, Munir A, Arafat W (2021) Psychological and sexual health during the COVID-19 pandemic in Egypt: are women suffering more? Sex Med 9(1):100295. https://doi.org/10.1016/j.esxm.2020.100295 (Epub 2021 Jan 9. PMID: 33434851; PMCID: PMC7794051)

8. Barqawi A, O’Donnell C, Kumar R, Koul H, Crawford ED (2005) Correlation between LUTS (AUA-SS) and erectile dysfunction (SHIM) in an age-matched racially diverse male population: data from the Prostate Cancer Awareness Week (PCAW). Int J Impot Res 17(4):370-374. https://doi.org/10.1038/sj.ijir.3901340 (PMID: 15889121)

9. Raisi-Estabragh Z, McCracken C, Bethell MS, Cooper J, Cooper C, Caulfield MJ, Munroe PB, Harvey NC, Petersen SE (2020) Greater risk of severe COVID-19 in Black, Asian and Minority Ethnic populations is not explained by cardiometabolic, socioeconomic or behavioural factors, or by $25(\mathrm{OH})$-vitamin D status: study of 1326 cases from the UK Biobank. J Public Health (Oxf) 42(3):451-460. https://doi.org/10.1093/pubmed/fdaa095 (PMID: 32556213; PMCID: PMC7449237)

10. Blick C, Ritchie RW, Sullivan ME (2016) Is erectile dysfunction an example of abnormal endothelial function? Curr Vasc Pharmacol 14(2):163-167. https://doi.org/10.2174/157016111466615 1202205950 (PMID: 26638795)

11. Giugliano F, Esposito K, Di Palo C, Ciotola M, Giugliano G, Marfella R, D'Armiento M, Giugliano D (2004) Erectile dysfunction associates with endothelial dysfunction and raised proinflammatory cytokine levels in obese men. J Endocrinol Invest 27(7):665669. https://doi.org/10.1007/BF03347500 (PMID: 15505991)
12. Smeda M, Chlopicki S (2020) Endothelial barrier integrity in COVID-19-dependent hyperinflammation: does the protective facet of platelet function matter? Cardiovasc Res 116(10):e118 e121. https://doi.org/10.1093/cvr/cvaa190 (PMID: 32707576; PMCID: PMC7432357)

13. Hu B, Huang S, Yin L (2021) The cytokine storm and COVID-19. J Med Virol 93(1):250-256. https://doi.org/10.1002/jmv.26232 (Epub 2020 Sep 30. PMID: 32592501; PMCID: PMC7361342)

14. Sansone A, Mollaioli D, Ciocca G, Limoncin E, Colonnello E, Vena W, Jannini EA (2021) Addressing male sexual and reproductive health in the wake of COVID-19 outbreak. J Endocrinol Invest 44(2):223-231. https://doi.org/10.1007/s40618-020-01350-1 (Epub 2020 Jul 13. PMID: 32661947; PMCID: PMC7355084)

15. Sansone A, Mollaioli D, Ciocca G, Colonnello E, Limoncin E, Balercia G, Jannini EA (2021) "Mask up to keep it up": preliminary evidence of the association between erectile dysfunction and COVID-19. Andrology 9(4):1053-1059. https://doi.org/10.1111/ andr.13003 (Epub 2021 Mar 30. PMID: 33742540; PMCID: PMC8250520)

16. Kresch E, Achua J, Saltzman R, Khodamoradi K, Arora H, Ibrahim E, Kryvenko ON, Almeida VW, Firdaus F, Hare JM, Ramasamy R (2021) COVID-19 Endothelial dysfunction can cause erectile dysfunction: histopathological, immunohistochemi$\mathrm{cal}$, and ultrastructural study of the human penis. World J Mens Health 39(3):466-469. https://doi.org/10.5534/wjmh.210055 (Epub 2021 May 7. PMID: 33988001; PMCID: PMC8255400)

17. Haghpanah A, Masjedi F, Alborzi S, Hosseinpour A, Dehghani A, Malekmakan L, Roozbeh J (2020) Potential mechanisms of SARS-CoV-2 action on male gonadal function and fertility: current status and future prospects. Andrologia 53(1):13883. https:// doi.org/10.1111/and.13883 (Epub Oct 27. PMID: 33108833; PMCID: PMC7645932)

18. Schulster M, Bernie AM, Ramasamy R (2016) The role of estradiol in male reproductive function. Asian J Androl May-Jun 18(3):435-440. https://doi.org/10.4103/1008-682X.173932. PMID:26908066;PMCID:PMC4854098

19. Shen Q, Xiao X, Aierken A, Yue W, Wu X, Liao M, Hua J (2020) The ACE2 expression in Sertoli cells and germ cells may cause male reproductive disorder after SARS-CoV-2 infection. J Cell Mol Med 24(16):9472-9477. https://doi.org/10.1111/jcmm.15541 (Epub 2020 Jun 28. PMID: 32594644; PMCID: PMC7361928)

20. Renu K, Subramaniam MD, Chakraborty R, Myakala H, Iyer M, Bharathi G, Siva K, Vellingiri B, Valsala Gopalakrishnan A (2020) The role of Interleukin-4 in COVID-19 associated male infertility - a hypothesis. J Reprod Immunol 2020(142):103213. https://doi.org/10.1016/j.jri.2020.103213 (Epub 2020 Sep 30. PMID: 33080435; PMCID: PMC7526609)

21. Chow SC, Shao J, Wang H (2008) Sample size calculations in clinical research, 2nd edn. Chapman \& Hall/CRC, Boca Raton

22. Levin KA (2006) Study design III: cross-sectional studies. Evid Based Dent 7(1):24-25

23. Jannini EA (2017) The interface of systems medicine and sexual medicine for facing non-communicable diseases in a genderdependent manner. Sex Med Rev 5(3):349-364. https://doi.org/10. 1016/j.sxmr.2017.04.002 (Epub 2017 Jun 7. PMID: 28596070)

24. Kloner RA (2008) Erectile dysfunction as a predictor of cardiovascular disease. Int J Impot Res 20(5):460-465. https://doi.org/ 10.1038/ijir.2008.20 (Epub 2008 May 15 PMID: 18480824)

25. Liu PP, Blet A, Smyth D, Li H (2020) The science underlying COVID-19: Implications for the cardiovascular system. Circulation 142(1):68-78. https://doi.org/10.1161/CIRCULATIONAHA. 120.047549 (Epub 2020 Apr 15 PMID: 32293910)

26. Xu Z, Shi L, Wang Y, Zhang J, Huang L, Zhang C, Liu S, Zhao P, Liu H, Zhu L, Tai Y, Bai C, Gao T, Song J, Xia P, Dong J, Zhao J, Wang FS (2020) Pathological findings of COVID-19 associated with acute respiratory distress syndrome. Lancet Respir Med 
8(4):420-422. https://doi.org/10.1016/S2213-2600(20)30076-X (Epub Feb 18. Erratum in: Lancet Respir Med. 2020 Feb 25; PMID: 32085846; PMCID: PMC7164771)

27. Madjid M, Safavi-Naeini P, Solomon SD, Vardeny O (2020) Potential effects of coronaviruses on the cardiovascular system: a review. JAMA Cardiol. 5(7):831-840. https://doi.org/10.1001/ jamacardio.2020.1286 (PMID: 32219363)

28. Patel KP, Vunnam SR, Patel PA, Krill KL, Korbitz PM, Gallagher JP, Suh JE, Vunnam RR (2020) Transmission of SARS-CoV-2: an update of current literature. Eur J Clin Microbiol Infect Dis 39(11):2005-2011. https://doi.org/10.1007/s10096-020-03961-1 (Epub 2020 Jul 7. PMID: 32638221; PMCID: PMC7339796)

29. Gkogkou E, Barnasas G, Vougas K, Trougakos IP (2020) Expression profiling meta-analysis of ACE2 and TMPRSS2, the putative anti-inflammatory receptor and priming protease of SARS-CoV-2 in human cells, and identification of putative modulators. Redox Biol 36:101615. https://doi.org/10.1016/j.redox.2020.101615 (Epub 2020 Jun 24. PMID: 32863223; PMCID: PMC7311357)

30. Hoffmann M, Kleine-Weber H, Schroeder S, Krüger N, Herrler T, Erichsen S, Schiergens TS, Herrler G, Wu NH, Nitsche A, Müller MA, Drosten C, Pöhlmann S (2020) SARS-CoV-2 cell entry depends on ACE2 and TMPRSS2 and is blocked by a clinically proven protease inhibitor. Cell 181(2):271-280.e8. https:// doi.org/10.1016/j.cell.2020.02.052 (Epub 2020 Mar 5. PMID: 32142651; PMCID: PMC7102627)

31. Rastrelli G, Di Stasi V, Inglese F, Beccaria M, Garuti M, Di Costanzo D, Spreafico F, Greco GF, Cervi G, Pecoriello A, Magini A, Todisco T, Cipriani S, Maseroli E, Corona G, Salonia A, Lenzi A, Maggi M, De Donno G, Vignozzi L (2021) Low testosterone levels predict clinical adverse outcomes in SARS-CoV-2 pneumonia patients. Andrology 9(1):88-98. https://doi.org/10.1111/ andr.12821 (Epub 2020 Jun 3. PMID: 32436355; PMCID: PMC7280645)

32. Isidori AM, Buvat J, Corona G, Goldstein I, Jannini EA, Lenzi A, Porst H, Salonia A, Traish AM, Maggi M (2014) A critical analysis of the role of testosterone in erectile function: from pathophysiology to treatment-a systematic review. Eur Urol 65(1):99-112. https://doi.org/10.1016/j.eururo.2013.08.048 (Epub 2013 Aug 29. PMID: 24050791)

33. Mohamad NV, Wong SK, Wan Hasan WN, Jolly JJ, Nur-Farhana MF, Ima-Nirwana S, Chin KY (2019) The relationship between circulating testosterone and inflammatory cytokines in men. Aging Male 22(2):129-140. https://doi.org/10.1080/13685538. 2018.1482487 (Epub 2018 Jun 21. PMID: 29925283)

34. Fang D, Peng J, Liao S, Tang Y, Cui W, Yuan Y, Wu D, Hu B, Wang R, Song W, Gao B, Jin L, Zhang Z (2021) An online questionnaire survey on the sexual life and sexual function of chinese adult men during the coronavirus disease 2019 epidemic. Sex Med 9(1):100293

35. Mollaioli D, Sansone A, Ciocca G, Limoncin E, Colonnello E, Di Lorenzo G, Jannini EA (2021) Benefits of sexual activity on psychological, relational, and sexual health during the COVID-19 breakout. J Sex Med 18(1):35-49. https://doi.org/10.1016/j.jsxm. 2020.10.008 (Epub 2020 Oct 23. PMID: 33234430; PMCID: PMC7584428)

Publisher's Note Springer Nature remains neutral with regard to jurisdictional claims in published maps and institutional affiliations. 